\title{
Evaluation of competing mortalities in oral squamous cell carcinoma patients treated with surgical and nonsurgical therapies
}

\author{
Ashwini Nareshchandra Walke1*, Tushar Vitthalrao Bhagat2 and Minal Chaudhary3 \\ ${ }^{1}$ MDS, Oral Pathologist, Riyadh, Saudi Arabia \\ ${ }^{2}$ MDS, Faculty \& Course Director for Clinical \& Preclinical Fixed Prosthodontics, Department of Prosthetics Dental sciences, College of Dentistry, Prince Sattam \\ Bin Abdulaziz University AlKarj, Riyadh, Kingdom of Saudi Arabia \\ ${ }^{3}$ Dean SPDC, Sawangi, Wardha, Riyadh, Kingdom of Saudi Arabia
}

\begin{abstract}
Oral squamous cell carcinoma (OSCC) is the sixth most common malignancy in the world. The incidence of oral carcinoma is more prevalent in males as they are twice more likely to develop oral carcinoma than women. $90 \%$ of people who develop oral carcinoma are those who use some sort of tobacco products. The etiology appears to be multifactorial and strongly related to lifestyle, mostly habits and diet. The five-year survival rate of oral carcinoma is about $50 \%$ which has not changed significantly in recent 50 years despite of advances in treatment modalities. The 'distinct reoccurrences, secondary malignancies, advance age and comorbid cardiopulmonary diseases are factors that predisposes patient to competing causes of death.
\end{abstract}

\section{Introduction}

Although the oral cavity carcinoma is substantially a global health concern besides that countries like India, where oral carcinoma is among top ten leading cause of death since 1991 [1,2]. The biggest mortality occurred in male during 2008 was due to oral cavity carcinoma and it was stated sixth most common cause of carcinoma occurrence [2]. The acknowledgment of survivors of head and neck carcinoma have augmented the morbidity and mortality compared with the healthy population that relates to treatment sequelae, coexisting pulmonary, cardiac, and liver diseases secondary to smoking and alcohol use, and second primary tumors [3-5].During early stage of oral cavity carcinoma surgical intervention or radiotherapy is often satisfactory remedy, whereas in advance stage violent remedies like surgery and post-operative adjuvant radiotherapy with or without chemotherapy is mandatory [3]. In past decades not more than 5-year survival rate has not improved appreciably though supplementary effective amalgamated multidiscipline remedies have implemented for tumor control and to improve quality of life in oral carcinoma patients [6]. Carcinoma cachexia, old age is the primary reason for death with oral cavity carcinoma patients, but comorbidities likewise employed a major effect on survivors. Even though locoregional and distant recurrence continues to be common events after combined-modality therapy, patients with oral cavity carcinoma are concurrently at risk for other events, including second malignancies and mortality because of antagonistic treatment effects or comorbid diseases [7].

Advanced age and comorbid cardiopulmonary diseases are factors that predispose patients to competing (non-cancerous) causes of death, while sepsis, organ failure, pulmonary embolism, surgical complications, or vascular disease remain common causes of treatment-related mortality.
Asia countries like India, after the diagnosis of carcinoma, the origin of competing comorbidities in oral cavity carcinoma seems to be associated with persistence of habit of betel quid chewing, smoking and alcohol consumption [1]. However along with mention factors, lack of finance, carcinoma cachexia, loss of follow up visits are also playing role in mortality rate, and these are recently found in SPDC, DMIMS, Wardha, Cancer Institution. In last decades, numerous studies have engrossed on analyzing the risk factors for competing events in head and neck carcinoma patients.

The primary aim of this retrospective study was to estimate the percentage of competing mortalities of who died after being treated for OSCC with surgical and combined therapy in DMIMS, Wardha institution, which is in an area endemic for betel quid chewing. And the objectives were to calculate the survival rate of patients who are treated with surgical and combined therapy. And also, to evaluate and analyze the causes of death in patients who did not survive for 5 years after treatment.

\section{Materials and Methods}

According to TNM stage reclassified American Joint Committee on Carcinoma staging system published in 2002, patients who had pathological stage I to IVB of oral cavity carcinoma and were treated with curative intent, either with radical surgery, radical surgery and adjuvant radiotherapy, radical surgery and adjuvant concurrent

${ }^{\star}$ Correspondence to: Ashwini Nareshchandra Walke, MDS, Oral Pathologist, Riyadh, Saudi Arabia, Tel: 091-9975771205; E-mail: ashwiniwalke2210@gmail.com

Key words: Reoccurrences, squamous cell carcinoma, tobacco

Received: March 15, 2018; Accepted: March 28, 2018; Published: March 30, 2018 
chemoradiotherapy, or primary concurrent chemoradiotherapy were eligible for this study. Patients with distant metastasis, or another concomitant active carcinoma were excluded from the study.

This was a retrospective study which was carried out in the Department of Oral Pathology and Microbiology, (SPDC). The addresses of patient were obtained from the archives of our institution.

The study consists of 120 patients, of age 30 to 75 years that were diagnosed cases of OSCC and treated with surgical and nonsurgical therapies from January 2008 to December 2011. The relative of the patient who were not survived are asked to fill up the questionnaires regarding causes of death. Format of the questionnaires given to the relatives was in Marathi and English language. The enrolled patients for the study were analyzed from a questionnaire and information concerning patient's cause of death which had recorded from close (blood relation) relatives. The cause of death was recorded as "disease" until every other suspected possibility was ruled out. Competing mortality was defined as death from any cause without experiencing any prior carcinoma event. Treatment related mortality included death from any cause within 1 months after treatment or death immediately after surgical complications, consistent with previously described methods [5]. The age, gender, primary site of disease, stage at diagnosis, treatment modality, exposure to smoking, alcohol and betel quid was common for every case. Statistical analyses were performed with the Statistical Package for the Social Sciences (SPSS), version 19.0. By using Pearson's chi-square ( $\chi 2)$ tests or Fisher's exact test (when the expected number per cell $<5$ ) to analyze the association between risk factors and the occurrence of competing mortality. Differences were considered significant when $P<0.05$. This study was approved by the Institutional ethics committee, DMIMS, Sawangi, Wardha.

\section{Results}

The age of patients during diagnosis was in between 30 to 75 years (mean, 52.5 years) and the dominant $59.2 \%$ gender was male for entire group of oral cavity carcinoma patients earlier to treatment. Over $55 \%$ of the enrolled patients had previously smoked, $75.8 \%$ addicted to alcohol, and $39.2 \%$ had a habit of betel quid (Table 1). After a median follow-up time of 32.1 months (range, 2.2 to 55.3 months), a total of 42 deaths occurred; 6 deaths (14.28\%) were due to carcinoma recurrence and progression, 6 deaths (14.28\%) were due to advanced age, while the other 25 patients died of competing (non-carcinoma) causes. Of those competing mortalities, 8 deaths $(19.04 \%)$ were due to carcinoma cachexia, 3 deaths $(7.14 \%)$ due to associated systemic illness (COPD). 6 deaths $(14.28 \%)$ due to continuation of habits, 5 deaths $(11.90 \%)$

Table 1. Demographic characteristic of all oral cavity carcinoma patients recorded before treatment.

\begin{tabular}{|c|c|}
\hline & Frequency \\
\hline No. Of patients & 120 \\
\hline Age, year (mean \pm sd) & 52.5 \\
\hline Gender & \\
\hline Male & 71 \\
\hline Female & 49 \\
\hline Stage & \\
\hline I & 51 \\
\hline Ii & 38 \\
\hline Iii & 18 \\
\hline Iva, b & 13 \\
\hline Exposure to smoking & $61(50.83 \%)$ \\
\hline Exposure to alcohol & $58(48.33 \%)$ \\
\hline Exposure to betel quid & $99(82.5 \%)$ \\
\hline
\end{tabular}

lack of finances, 3 deaths (7.14\%) due to patients lost follow up visits, 1 death is due to treatment related complication during surgery (2.38). Of treatment related mortality, $4(9.52 \%)$ patients received concurrent chemoradiotherapy. Pretreatment poor performance status (performance status $>1$ ) was the only factor significantly correlated with the occurrence of competing mortality as determined by chi-square $\left(\chi^{2}\right)$ tests $(\mathrm{P}<0.01) .78$ patients were in good condition after treated with surgical and nonsurgical therapies, 42 patients were reported death, 8 patients were no more because of carcinoma cachexia, 6 patients were continued the habit, 6 were died because of old age, 6 patients had got recurrence of the carcinoma, 5 patients not underwent any treatment as lack of finance, 4 patients choose not to receive further treatment (chemo-radiotherapy and chemo- radiotherapy plus surgery), 3 patients died because of associated with systemic illness, 3 patients lost to follow-up immediately after the completion of treatment, 1 patients died because of treatment related complication during surgery.

\section{Discussion}

Combinations of the multidiscipline approach, including surgery and radiotherapy (with or without chemotherapy) are widely used in cases of advanced oral cavity carcinoma, which improve tumor control, survival rates [8]. In our study, total of 42 deaths reported next to median follow-up time of 32.1 months. Primary reason of death was cancer cachexia that is a wasting syndrome characterized particularly by anemia, loss of body weight, anorexia and asthenia. Consequences of cachexia are state of active inflammation by tumor-derived factors TNF- $\alpha$, IL-1 and IL-6.TNF-alpha and the tumor factor proteolysisinducing factor causing skeletal muscle atrophy. And the abnormal host reaction to these features result in a catabolic state [9]. A longitudinal study has shown that $2.5 \mathrm{~kg}$ weight change over $6-8$ weeks is sufficient to produce significant changes in performance status.

Recently it has been proposed to include additional features to identify the cachexia syndrome such as involuntary weight loss, reduced muscle mass, anorexia, and biochemical alterations (-Reactive Protein (CRP), albumin, hemoglobin) [9]. A meta-analysis investigating the outcome of dietary counseling on clinical outcomes in carcinoma patients revealed a trend suggesting an improvement in quality of life [10].

The prevalence of second primary tumors associated to the site of primary, stage of disease (stage II more than I), and smoking status. Many studies have also reported that the amount of tobacco use and smoking continuation correlate with the risk of a second primary tumor [2].

Almost $30-50 \%$ of second primary tumors progress in the head and neck. Because these tumors are uniformly squamous and despite the employment of strict criteria, in many cases it is uncertain whether the presumed second primaries represent local recurrences [11].

For chemotherapy-related death in many carcinoma patients, has been found to be correlated with the risk of severe infection during multimodality treatment.

Older patients have higher rates of hematologic toxicity and treatment-related death than younger patients during adjuvant chemotherapy. In our report, 3 patients who died of not receiving complete treatment protocol suggested for carcinoma therapy (2 received concurrent chemoradiotherapy and 1 received surgery plus concurrent chemo radiotherapy) [11].

Treatment-related complications is also the cause of death other than disease during treatment or soon after treatment completion. In 
our study death due to pneumonia occurs in one patient within one month after surgery. Advanced age and comorbid cardiopulmonary diseases are factors that predispose patients to competing causes of death. Our study showed that liver cirrhosis is the most common factor contributing to competing mortality other than treatment-related mortality, the main etiology of liver cirrhosis includes alcohol (Table 2). Alcoholism is the main etiology of liver cirrhosis and one of the causative agents in oral cavity carcinoma. More than $70 \%$ of patients in our study exposed to alcohol prior to the carcinoma diagnosis in our study. Therefore, Physicians should not only focus on the treatment efficacy of oral cavity carcinoma but also notice the development and progression of liver cirrhosis.

There are one more critical observations that the patients continue his habit of betel nut chewing and alcohol consumption even after treated for the carcinoma [11]. Second, betel quid chewing is prevalent in our area. Of particular concern is the fact that approximately $85 \%$ of oral cavity carcinoma patients are habitual users of betel quid, which is strongly associated with adult metabolic syndrome. And oral mucosa changes including mucosa degeneration, leukoplakia and OSMF. Oral mucosal change decreases local immunity, subsequently leading to systemic infection during courses of intensive treatment; this makes patients considerably more vulnerable to treatment related morbidity and mortality. Betel chewing is considered hepatotoxic and hepatoma carcinogenic, due to the high concentration of aflatoxin B1 in areca catechu nuts, the high concentration of safrole $\mathrm{P}$ in betel plants. Clinically, Hsiao et al., Wet al. reported that individuals with elevated betel quid chewing had higher risks for liver cirrhosis [11].

Another two new causes of mortality found in our study, one in which the patients lost further follow up visits after completion of treatment and the second, due to lack of finances the poor patients did not turn up for further management. These two causes may be correlated with each other. Despite of many programs run by the government for carcinoma patients still there is poor awareness among the people regarding carcinoma.

Our study result showed that carcinoma cachexia, lack of finances, continuation of habit is common factor contributing to competing mortality, which is somewhat different from previous studies.

Despite limited sample size $(\mathrm{N}=120)$, and a relatively short followup time (median $=32.1$ months) in our study, this serious comorbidity, liver cirrhosis, still deserves ongoing focus in the treatment of oral cavity carcinoma. Abstinence from substance use such as alcohol, smoking and betel quid should be suggested in patients with oral cavity carcinoma to reduce further competing causes of death.

Furthermore, health care policy makers and providers in our institute need to develop policies and programs targeting oral cavity

Table 2. Causes of competing mortality $(\mathrm{N}=42)$

\begin{tabular}{|l|c|c|}
\hline Reason & No. Of death & Percentage \\
\hline Carcinoma cachexia & 8 & $19.04 \%$ \\
\hline Recurrence & 6 & $14.28 \%$ \\
\hline Old age & 6 & $14.28 \%$ \\
\hline $\begin{array}{l}\text { Continuation of habit of betel nut and alcohol } \\
\text { consumption }\end{array}$ & 6 & $14.28 \%$ \\
\hline Lack of finance & 5 & $11.90 \%$ \\
\hline Incomplete treatment chemotherapy or surgery & 4 & $9.52 \%$ \\
\hline $\begin{array}{l}\text { Associated with systemic illness } \\
\text { cardiopulmonary or liver disease) }\end{array}$ & 3 & $7.14 \%$ \\
\hline Patient lost follow up & 3 & $7.14 \%$ \\
\hline Treatment related complication & 1 & $2.38 \%$ \\
\hline
\end{tabular}

carcinoma prevention, which in part emphasize measures to eliminate the habit of betel nut chewing.

The survival rate up to $50 \%$ for five years in patients of OSCC has been a challenge to all concerned. On detailed analysis we found that the competing comoratalities of OSCC is range from, recurrence, continuation of habit to carcinoma cachexia.

We divided these causes into two categories:

1.Factors in which interception would be beneficial

\section{Factors in which interception would not be feasible}

Carcinoma cachexia, cardiopulmonary disorders, continuation of habit and lack of finances are the factors included in the first category. Collectively, these contribute up to $35 \%$ deaths after treatment. We believe that interception to address these factors would be beneficial. The interception would in the form of psychological counseling, proper access to medical care and financial assistance.

This would result in appreciable increased in 5 years survival of patients of OSCC.

Finally, oncologists need to be adept at assessing patient physiological and functional capacity associated with substance abuse and related comorbidities, in order to provide safe and efficacious oral cavity carcinoma treatment [11].

\section{Conclusion}

The responsibility of the surgeon and treating physician does not stop with the surgery in the patients with OSCC. The ultimate goal of any treatment is to provide a maximum number of disease free life, if not cure. This study revealed that the causes of death in OSCC patients post treated could be attributed to carcinoma cachexia, continuation of habit and lack of finances. Addressing these factors would play a crucial role in providing a long-term disease-free survival of patients with OSCC.

\section{Acknowledgement}

The authors are thankful to all the members of department of oral pathology and microbiology for providing all the needed help.

\section{References}

1. Murthy V, Agarwal J, Laskar SG, Gupta T, Budrukkar A, et al. (2010) Analysis of prognostic factors in 1180 patients with oral cavity primary carcinoma treated with definitive or adjuvant radiotherapy. Journal of Cancer Research and Therapeutics 6: 282-289. [Crossref]

2. Yeole BB, Sankaranarayanan R, Sunny L, Swaminathan R, Parkin D (2000) Survival from head and neck carcinoma in Mumbai (Bombay), India. Cancer 89: 437-444. [Crossref]

3. Chang P-H, Yeh K-Y, Huang J-S, Huang Y-M, Chang Y-S (2013) Competing Causes of Death in Patients with Oral Cavity Carcinoma in a Single Carcinoma Center in Taiwan. J Cancer Res Pract 29: 14-21.

4. Bell RB, Kademani D, Homer L, Dierks EJ, Potter BE (2007) Tongue carcinoma: Is there a difference in survival compared with other subsites in the oral cavity? $J$ Oral Maxillofac Surg 65: 229-236. [Crossref]

5. Argiris A, Brockstein BE, Haraf DJ, Stenson KM, Mittal BB, et al. (2004) Competing causes of death and second primary tumors in patients with locoregionally advanced head and neck carcinoma treated with chemoradiotherapy. Clin Carcinoma Res 10: 1956-1962. [Crossref]

6. Zhang H, Dziegielewski PT, Biron VL, Szudek J, Al-Qahatani KH, et al. (2013) Survival outcomes of patients with advanced oral cavity squamous cell carcinoma treated with multimodal therapy: a multi-institutional analysis. J Otolaryngol Head Neck Surg 42: 30. [Crossref]

7. Wutzl A, Ploder O, Kermer C, Millesi W, Ewers R (2007) Mortality and causes of death after multimodality treatment for advanced oral and oropharyngeal carcinoma. $J$ Oral Maxillofac Surg 65: 255-260. [Crossref] 
Walke AN (2018) Evaluation of competing mortalities in oral squamous cell carcinoma patients treated with surgical and nonsurgical therapies

8. Haddad RI, Shin DM (2008) Recent advances in head and neck carcinoma. $N$ Engl J Med 359: 1143-1154. [Crossref]

9. Chang P-H, Yeh K-Y, Huang J-S, Lai C-H, Wu T-H, et al. (2013) Pretreatment performance status and nutrition are associated with early mortality of locally advanced head and neck carcinoma patients undergoing concurrent chemoradiation. Eur Arch Otorhinolaryngol. 270: 1909-1915. [Crossref]
10. Gomez H, Hidalgo M, Casanova L, Colomer R, Pen D, et al. (1998) Risk factors for treatment-related death in elderly patients with aggressive non-Hodgkin's lymphoma: results of a multivariate analysis. J Clin Oncol 16: 2065-2069. [Crossref]

11. Parkin DM, Pisani P, Ferlay J (1999) Estimates of the worldwide incidence of 25 major carcinomas in 1990. Int J Carcinoma 80: 827-841. [Crossref]

Copyright: $@ 02018$ Walke AN. This is an open-access article distributed under the terms of the Creative Commons Attribution License, which permits unrestricted use, distribution, and reproduction in any medium, provided the original author and source are credited. 